\title{
Bayesian Network Based Fault Prognosis via Bond Graph Modeling of High-Speed Railway Traction Device
}

\author{
Yunkai Wu, ${ }^{1,2}$ Bin Jiang, ${ }^{1,2}$ Ningyun Lu, ${ }^{1,2}$ and Yang Zhou ${ }^{3}$ \\ ${ }^{1}$ College of Automation Engineering, Nanjing University of Aeronautics and Astronautics, Nanjing 210016, China \\ ${ }^{2}$ Jiangsu Key Laboratory of Internet of Things and Control Technologies, Nanjing University of Aeronautics and Astronautics, \\ Nanjing 210016, China \\ ${ }^{3}$ School of Computer Science and Engineering, Jiangsu University of Science and Technology, Zhenjiang 212003, China
}

Correspondence should be addressed to Bin Jiang; binjiang@nuaa.edu.cn

Received 5 September 2014; Accepted 25 December 2014

Academic Editor: Peng Shi

Copyright (C) 2015 Yunkai Wu et al. This is an open access article distributed under the Creative Commons Attribution License, which permits unrestricted use, distribution, and reproduction in any medium, provided the original work is properly cited.

Reliability of the traction system is of critical importance to the safety of CRH (China Railway High-speed) high-speed train. To investigate fault propagation mechanism and predict the probabilities of component-level faults accurately for a high-speed railway traction system, a fault prognosis approach via Bayesian network and bond graph modeling techniques is proposed. The inherent structure of a railway traction system is represented by bond graph model, based on which a multilayer Bayesian network is developed for fault propagation analysis and fault prediction. For complete and incomplete data sets, two different parameter learning algorithms such as Bayesian estimation and expectation maximization (EM) algorithm are adopted to determine the conditional probability table of the Bayesian network. The proposed prognosis approach using Pearl's polytree propagation algorithm for joint probability reasoning can predict the failure probabilities of leaf nodes based on the current status of root nodes. Verification results in a high-speed railway traction simulation system can demonstrate the effectiveness of the proposed approach.

\section{Introduction}

CRH (China Railway High-speed) high-speed train traction system is a complex electromechanical coupling system, which consists of a lot of electrical and mechanical devices, such as pantograph, traction transformers, traction converters, and traction motors. Along with the growth of running time, some components in a traction system like IGBTs (insulated gate bipolar transistors) and diodes will degrade with age. These fatigued components are likely to have various abrupt faults such as short-circuit or open-circuit faults, which definitely increase the risk of serious accidents in the entire railway. Thus, fault prognosis is urgently demanded in high-speed railway traction systems.

Due to the complex structures and behaviors of electromechanical coupling traction systems, it is difficult to describe the causalities accurately through analytical models, which limits the application of the existing analytical model based fault prognosis methods [1]. Instead, the data-driven statistical model based prognosis methods, especially the Bayesian networks, have become the mainstream [2-7]. However, constructing an accurate Bayesian network structure is a big challenge in practice. Some scholars have proposed approaches to learn the Bayesian network structure from data $[4,5]$. Due to the reason that the accuracy of the learned Bayesian network is largely affected by the richness of the data and the prior knowledge of the network ordering, few attractive results have been obtained, according to which an idea of constructing Bayesian network via bond graph model is proposed in this paper.

Bond graph modeling has been widely applied to lots of engineering fields for modeling various dynamic systems. It is particularly popular for modeling electromechanical coupling systems. In bond graphs, different elements that belong to different energy domains (such as mechanical, electrical, and electromagnetic domains) can be described by the same model structure with uniform modeling language. Bond graph theory and its recent applications have been 


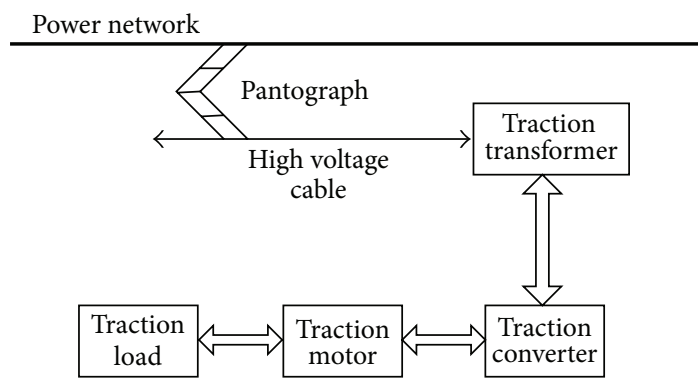

FIgURE 1: Schematic diagram of $\mathrm{CRH}_{5}$ traction system.

summarized in [8-10]. Due to its capability in describing the causality of a complex system, bond graph has been investigated in modeling of high-speed railway vehicles in recent years [11-13]. The bond graph model of a squirrel cage induction motor is introduced in [14], which contributes to the bond graph modeling of high-speed train traction device. Model based [15-17] and data-driven based [18, 19] FDD (fault detection and diagnosis) approaches, especially the bond graph based methods [20-25], are becoming hot research topics. However, the causal relationships in a bond graph that consist of important information for reasoning are seldom used in fault prognosis domain directly.

This paper proposes a general procedure for constructing a Bayesian network structure on the basis of bond graph modeling for fault prognosis of high-speed train traction device. The causal relationships revealed by the bond graph model are combined with the reasoning capacity of the Bayesian network. For complete and incomplete data sets, Bayesian estimation and expectation maximization (EM) algorithm are adopted, respectively, to determine the conditional probability table of the Bayesian network. Pearl's polytree propagation algorithm is used for joint probability reasoning. The failure probabilities of the other leaf nodes are determined by the current status of root nodes. The simulation results on $\mathrm{CRH}_{5}$ traction system can verify the effectiveness of the proposed approach.

\section{Modeling of $\mathrm{CRH}_{5}$ Traction Device}

2.1. Railway Traction System. $\mathrm{CRH}_{5}$ high-speed trains are providing convenient public transportation among major cities in China. According to [26], the $\mathrm{CRH}_{5}$ traction system is made up of pantograph (model: DSA250), vacuum circuit breaker (model: 22CBNG), traction transformer (electrical standard: IEC 60310), traction converter (model: YGN2Q213), traction motor (model: YJ87A, three-phase squirrel cage induction motor), and other components. An AC-DC-AC driving method is adopted, as shown in Figure 1, where $25 \mathrm{kV}$ HVAC (high voltage alternating current) is transformed into $1700 \mathrm{~V}$ AC (alternating current) by the traction transformer through pantograph; then, the converter (AC-DC-AC) outputs three-phase AC with controllable voltage and frequency for the traction motors.

For simplicity, the traction system consisting of two sets of inverters and induction motors is studied in this paper.
The three-phase inverter bridge circuits can realize VVVF (variable velocity variable frequency) drive of the three-phase ACIMs (alternating current induction motors). The inverter circuit, as shown in Figure 2, uses IGBTs as switch elements in its main circuit and control system design, where the voltagespace vector control scheme is adopted in the inverter control circuit.

Open-circuit fault and short-circuit fault are two kinds of common faults in traction inverters. Switch-on failure of the transistors and breakdown of the motor phase can cause open-circuit faults, which will increase torque pulsations, copper losses or reduce mean torque and efficiency. Switchoff failure of the transistors and ground of the phase terminals can cause short-circuit faults, which will bring overload burning of the stator and rotor circuits.

\subsection{Bond Graph Modeling}

2.2.1. Bond Graph: Basis. Electromechanical systems are governed by many effects issued from different physical phenomena and various technological components. Bond graph, a unified and multidomain modeling and simulation approach, is well suited for such systems. Bond graph provides possibilities for both structural and behavioral system analysis [27]. According to [28], power variable is the product of effort (represented by $e$ ) and flow (represented by $f$ ), where effort variable represents force, voltage, or pressure and flow variable represents current, flow, or velocity. Capacitance $(C)$, inertias $(I)$, resistances $(R)$, sources $\left\{S_{e}, S_{f}\right\}$, gyrator (GY), transformer (TF), and junctions 0,1 are generic bond graph components, which can be classified as 1-port components $\left\{S_{e}, S_{f}, I, R, C\right\}, 2$-port components $\{\mathrm{TF}, \mathrm{GY}\}$, and multipleports components $\{1,0\}$. These bond graph components are connected by a set of bonds represented by half arrows indicating positive energy flow from one variable to another. For each bond, an effort and a flow variable were remarked to describe the signals of the bond graph components connected to the bonds. The generic components and bond connections form the structure of bond graph model, where a set of relations called constitutive relations is used to describe the behavior of each component [29].

Besides the modeling capability for electromechanical systems, bond graph approach can derive equations or information from the graph itself by using a concept called the causality. In bond graphs, a stroke is marked at one end of each bond, which indicates the direction of an effort or flow signal. Users can derive the relations or analytical expressions between system variables to understand how fault signals propagate in the system.

2.2.2. Equivalent Circuits for $\mathrm{CRH}_{5}$ Traction Devices. The converter in $\mathrm{CRH}_{5}$ unit consists of two sets of rectifiers, two sets of inverters, one set of traction control device, and cooling system. Each traction motor is controlled by one set of inverters, whose equivalent circuit is shown in Figure 3. The three-phase AC motors can be equivalent to a series circuit of resistances, inductances, and counter electromotive force, where the effect of the counter electromotive force 
REO

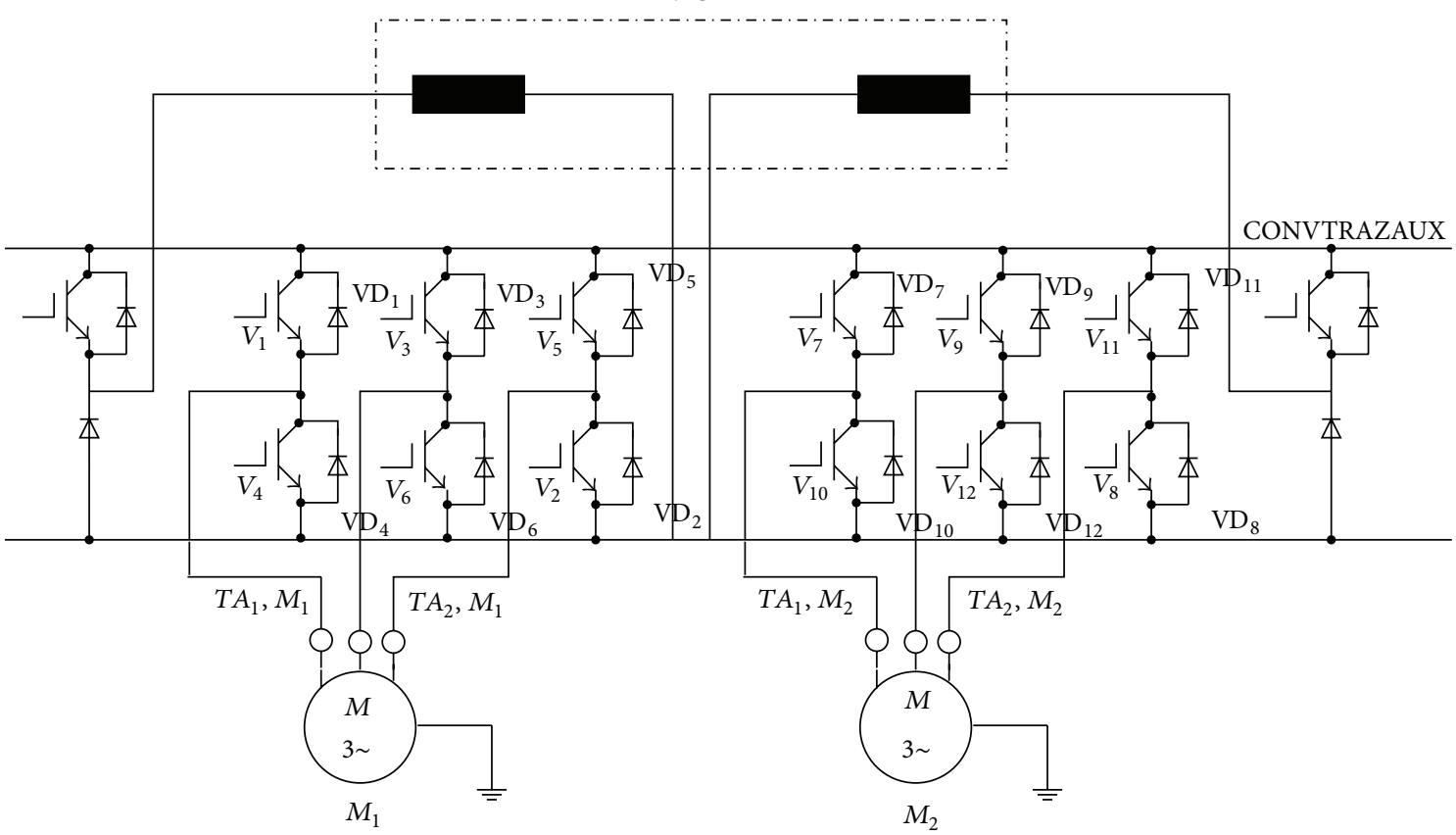

FIGURE 2: Schematic diagram of $\mathrm{CRH}_{5}$ inverter.

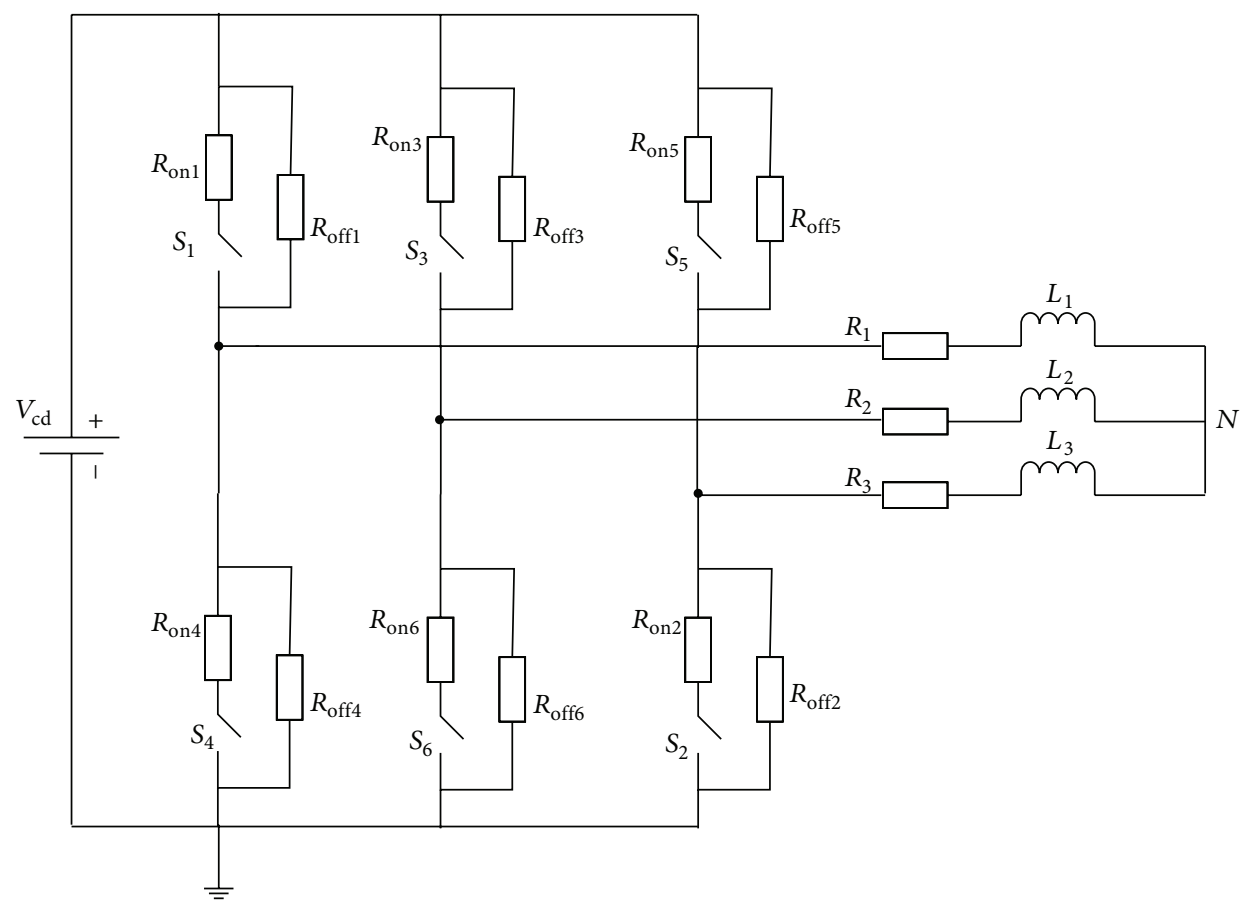

FIGURE 3: Equivalent circuit of the inverter and three-phase AC motor.

can be neglected when the load side is unloaded. For bond graph modeling of the inverter part, IGBT and diode circuit can be simplified by a switch model consisting of a turn-on resistance $R_{\text {on }}$ with low resistor value, a turn-off resistance $R_{\text {off }}$ with high resistor value, and an ideal switch $S$. In Figure 3 , the series connecting $R_{\text {onl }}$ and $S_{1}$ is the equivalent circuit of IGBT $V_{1}, R_{\text {offl }}$ is the equivalent circuit of diode $\mathrm{VD}_{1}$, and so on. Different equivalent resistances $\left(R_{\mathrm{eq}}\right)$ corresponding to different status can be calculated as follows:

$$
R_{\mathrm{eq}}= \begin{cases}R_{\mathrm{off}} & \text { for } S \text { turned off } \\ \frac{R_{\mathrm{on}} R_{\mathrm{off}}}{R_{\mathrm{on}}+R_{\mathrm{off}}} \approx R_{\mathrm{on}} & \text { for } S \text { turned on } .\end{cases}
$$




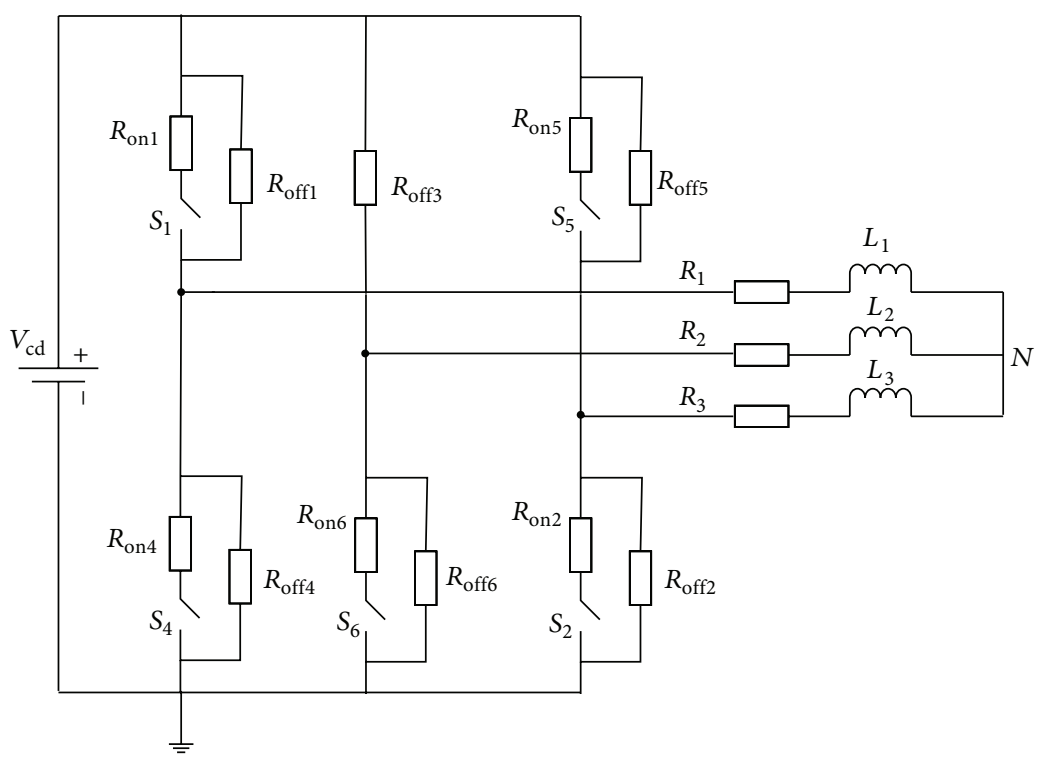

FIGURE 4: Equivalent circuit for open-circuit fault.

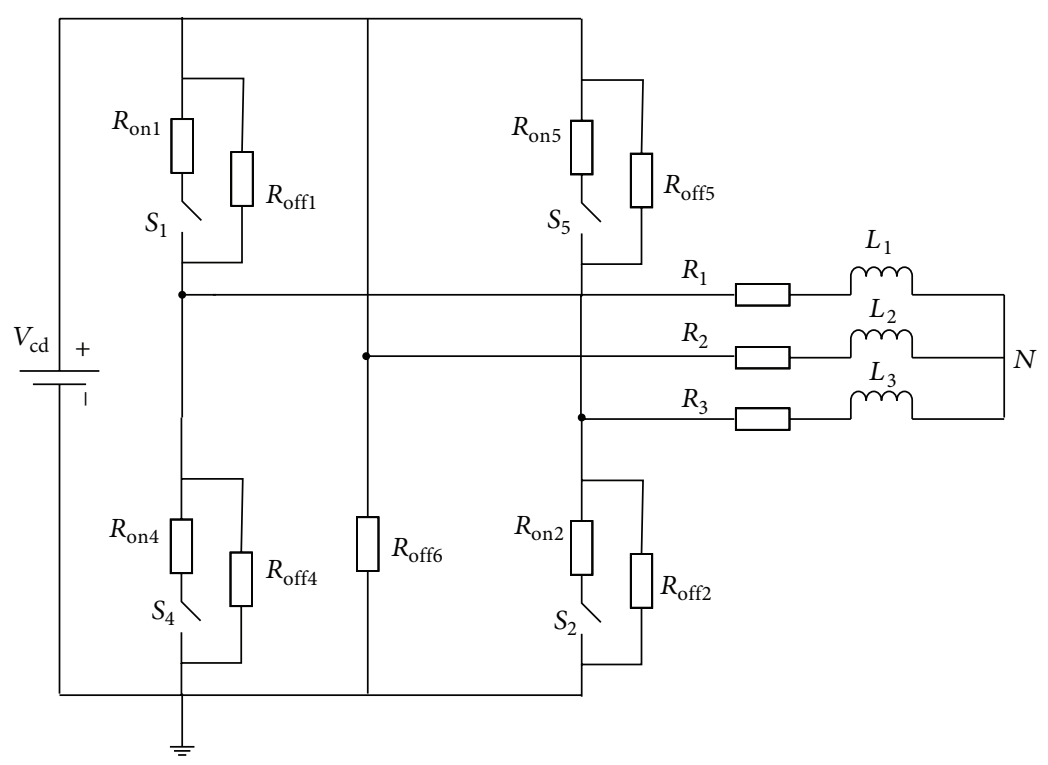

FIGURE 5: Equivalent circuit for short-circuit fault.

The final equivalent circuit of the inverter and three-phase AC motors for open-circuit fault and short-circuit fault status are shown in Figures 4 and 5, respectively.

2.2.3. Bond Graph Modeling of Traction System. According to [28], the main difficulty in bond graph modeling of power switching device is how to describe the discrete system dynamics. So far, many bond graph based modeling approaches for power switching device have been proposed, such as enumeration method, Petri Net method, and MTF (multitransformer) method. MTF method is the most popular method, where modulus $m=0$ or $m=1$ represents the status (ON or OFF) of power switching devices. As shown in Figure 6, $S_{1}-S_{12}$ and $R_{\text {offl }}-R_{\text {off12 }}$ represent switching tubes $V_{1}-V_{12}$ and diodes $\mathrm{VD}_{1}-\mathrm{VD}_{12}$, respectively, in the inverter circuit. In bond graph modeling of the three-phase AC motors, the resistance $R_{e}$ and the winding $L_{e}$ determine the behavior of the stator circuit; the resistance $R_{p}$ and the winding $L_{p}$ indicate the losses of hysteresis and the magnetic flux losses in the stator and rotor circuit; the resistance $R_{r}$ and the winding $L_{r}$ determine the rotor circuit behavior. The mechanical power generated by each phase is modeled by the "MGY" ports. The rotational mechanical power is added to the " 1 " junction, which can be applied to the motor shaft modeling by the inertia port " $I$ " with parameter " $J$." The friction losses are modeled by the resistance port " $R$ " with parameter " $\mu$." 


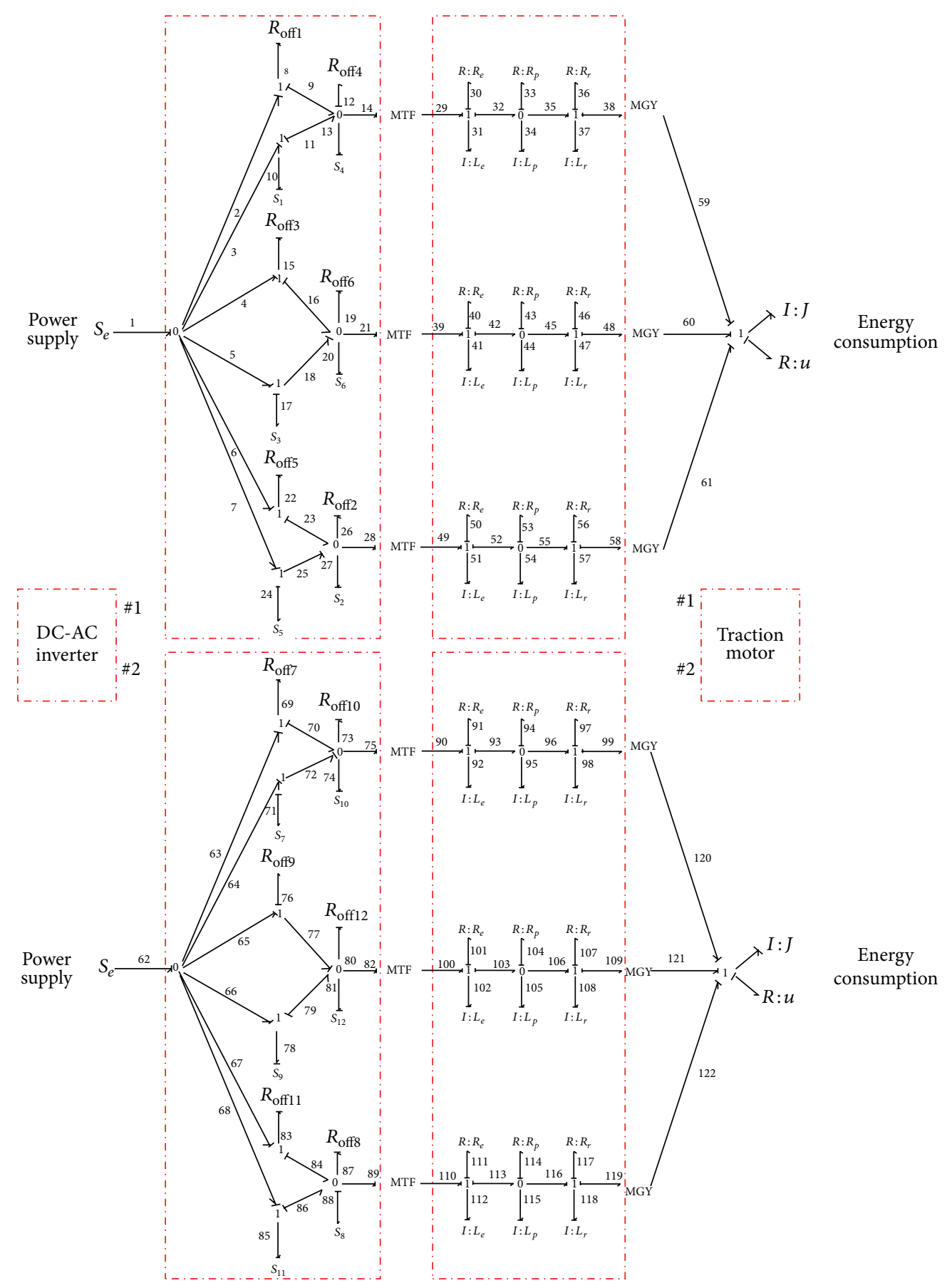

FIgURE 6: Bond graph modeling of $\mathrm{CRH}_{5}$ inverter and three-phase $\mathrm{AC}$ motor.

Open-circuit fault and short-circuit fault are two kinds of common faults in inverter circuits. According to circuit analysis, the features of the collector-emitter average voltage of the switching tube are the same when an open-circuit fault happens on $V_{1}, V_{3}$, or $V_{5}$ or a short-circuit fault happens on $V_{2}, V_{4}$, or $V_{6}$. Aiming at accurate fault location, define the behaviors of $R_{\text {on }}$ and $R_{\text {off }}$ as follows: the value of $R_{\text {on }}$ increases rapidly when an open-circuit fault happens; the value of $R_{\text {off }}$ decreases rapidly when a short-circuit fault happens. In Table $1, e_{10}, e_{13}, e_{17}, e_{20}, e_{24}$, and $e_{27}$ represent the collector-emitter average voltages of the bonds numbers 10 , $13,17,20,24$, and 27 , respectively. " 0 " represents the nominal value of the collector-emitter average voltage; "+" represents the increase of collector-emitter voltage; "-" represents the decrease of collector-emitter voltage.

According to the equivalent circuit shown in Figures 4 and 5, Figure 7(a) shows the bond graph of B-phase circuit in number 1 inverter, where IGBT $V_{3}$ has an open-circuit fault. Figure 7(b) shows the bond graph of B-phase circuit in number 1 inverter, where IGBT $V_{3}$ has a short-circuit fault. 
TABLE 1: Common faults in the bond graph model of $\mathrm{CRH}_{5}$ traction system.

\begin{tabular}{|c|c|c|c|c|c|c|c|}
\hline Fault & Description & $e_{10}$ & $e_{13}$ & $e_{17}$ & $e_{20}$ & $e_{24}$ & $e_{27}$ \\
\hline$F_{1}$ & Open-circuit fault $\left(S_{1}\right)$ or short-circuit fault $\left(S_{4}\right)$ & + & - & 0 & 0 & 0 & 0 \\
\hline$F_{2}$ & Short-circuit fault $\left(S_{1}\right)$ or open-circuit fault $\left(S_{4}\right)$ & - & + & 0 & 0 & 0 & 0 \\
\hline$F_{3}$ & Open-circuit fault $\left(S_{3}\right)$ or short-circuit fault $\left(S_{6}\right)$ & 0 & 0 & + & - & 0 & 0 \\
\hline$F_{4}$ & Short-circuit fault $\left(S_{3}\right)$ or open-circuit fault $\left(S_{6}\right)$ & 0 & 0 & - & + & 0 & 0 \\
\hline$F_{5}$ & Open-circuit fault $\left(S_{5}\right)$ or short-circuit fault $\left(S_{2}\right)$ & 0 & 0 & 0 & 0 & + & - \\
\hline$F_{6}$ & Short-circuit fault $\left(S_{5}\right)$ or open-circuit fault $\left(S_{2}\right)$ & 0 & 0 & 0 & 0 & - & + \\
\hline
\end{tabular}

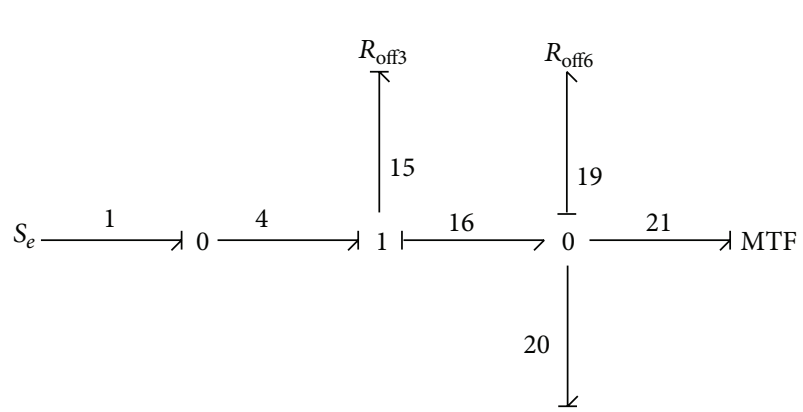

(a)

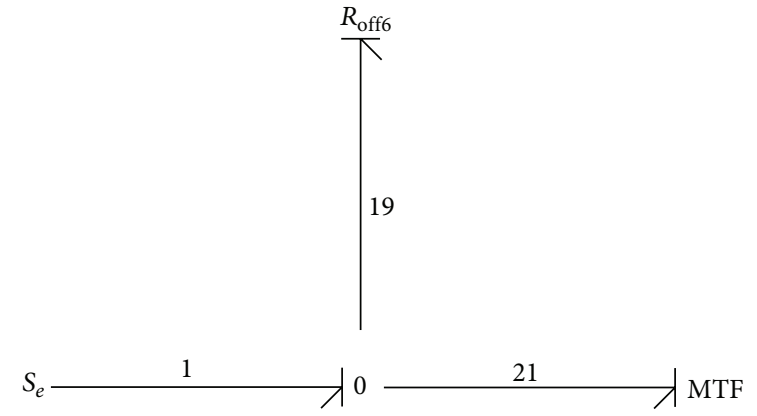

(b)

FIGURE 7: Bond graph modeling of B-phase inverter circuit in fault.

\section{Fault Prognosis Based on Bayesian Network}

3.1. Key Idea. Since the three-phase circuits in the inverter are exactly the same, B-phase circuit is shown as an example to study the fault prognosis in this paper. Figure 8 illustrates the fault prognosis mechanism using the Bayesian network of B-phase circuit to predict the fault probability of the stator or rotor circuit. For offline implementation, the bond graph model of $\mathrm{CRH}_{5}$ inverter and three-phase AC motor are used to construct a Bayesian network for fault prognosis. The Bayesian network based fault prognosis module is activated when the structure of predictive Bayesian network is obtained and the conditional probability distributions are acquired. For online implementation, evidence extracted from the system measurements can propagate through the network when the probability distributions for each variable are inferred.

\subsection{From Bond Graph to Bayesian Network}

3.2.1. Bayesian Network. Bayesian network is a directed acyclic graph (DAG), where nodes represent the random variables. The directed edges leading from cause variables to effect variables represent the causal relations. The measurements are donated by conditional probabilities between nodes and father-nodes. According to [30], prior probabilities need to be specified for root nodes, while conditional probability distributions (CPDs) are specified for nonroot nodes. The edges in the Bayesian network represent the joint probability distributions which can be defined as

$$
P(x)=\prod_{i=1}^{n} P\left(x_{i} \mid \text { parents }\left(x_{i}\right)\right)
$$

where parents $\left(x_{i}\right)$ is the parent set of node $x_{i}$. The equation above, known as a chain rule indicating the joint probability distribution of all variables in the Bayesian network, is the product of each variable's probabilities when its parents' values are given. The probability distribution of each variable or partial variable can be obtained by Bayesian network inference when other variables are known.

A Bayesian network comprises two parts $B=\langle G, \Theta\rangle$, where $G$ is a DAG conveying the direct dependence relationships within the data set, while $\Theta$ is the CPD of each variable. Assume that $\prod_{x_{i}}$ represents the set of direct parents of $x_{i}$ in G. $\Theta$ contains a parameter $\theta_{x_{i} \mid \prod_{x_{i}}}=P_{B}\left(x_{i} \mid \prod_{x_{i}}\right)$ for each $x_{i}$, such that the network $B$ can represent the following joint probability distribution [31]:

$$
P_{B}\left(x_{1}, x_{2}, \ldots, x_{n}\right)=\prod_{i=1}^{n} P_{B}\left(x_{i} \mid \prod_{x_{i}}\right)=\prod_{i=1}^{n} \theta_{x_{i} \mid \prod_{x_{i}}} .
$$

3.2.2. Bond Graph Based Causality Analysis. The consistent causality description facilitates FDI or fault prognosis design because it is helpful for analyzing the fault propagation in the whole system. Causal path, a graphic representation based on the concept of causality, shows the causal relationships among the system variables. " 0 " and " 1 " controlled junctions are the multiple-ports components in bond graph based modeling. According to the definition of flow variable and effort variable, " 1 " junction indicates that every flow variable of the bonds connected to the junction equals the other, where the input variable is defined as the flow variable of the connected bond that has no causal stroke assigned at the junction. Similarly, a " 0 " junction indicates that each effort variable of the adjacently connected bonds equals the other, 


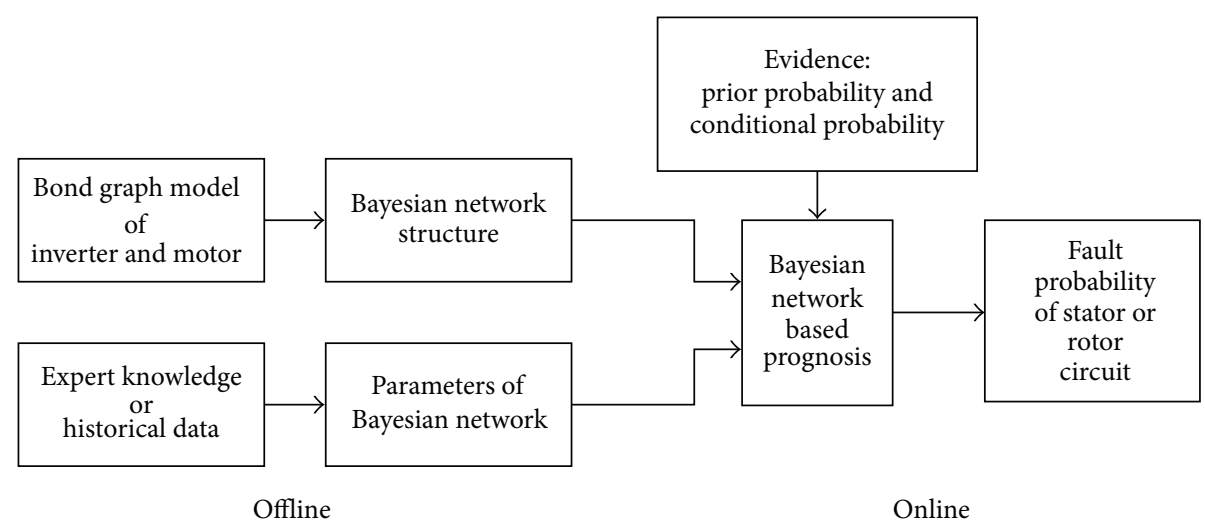

FIGURE 8: Overview of the fault prognosis based on Bayesian network.

where the input variable is defined as the effort variable of the connected bond that has a causal stroke assigned at the "0" junction. The storage elements are usually assigned preferred derivative causality [29].

The bond graph modeling implies the state equations describing the system dynamics. The causal relationships and power transfer between each component can be obtained clearly through the state equations coming from the bond graph. List the causal relationships of B-phase circuit in number 1 inverter as follows:

$$
\begin{aligned}
& e_{15}=e_{1}-e_{16}, \\
& e_{19}=e_{16}=e_{18}=e_{20}=e_{21}, \\
& f_{15}=\frac{e_{15}}{R_{\text {off } 3}}, \\
& e_{40}=R_{e} f_{40}, \\
& e_{17}=e_{1}-e_{18}, \\
& f_{41}=\frac{1}{L_{e}} \int e_{41} d t, \\
& f_{17}=\frac{m_{3}^{2}}{R_{\text {on } 3}} e_{17}, \\
& e_{43}=R_{p} f_{43}, \\
& e_{19}=R_{\text {off } 6} f_{19}, \\
& f_{44}=\frac{1}{L_{p}} \int e_{44} d t, \\
& f_{20}=\frac{m_{6}^{2}}{R_{\text {on } 6}} e_{20},
\end{aligned}
$$

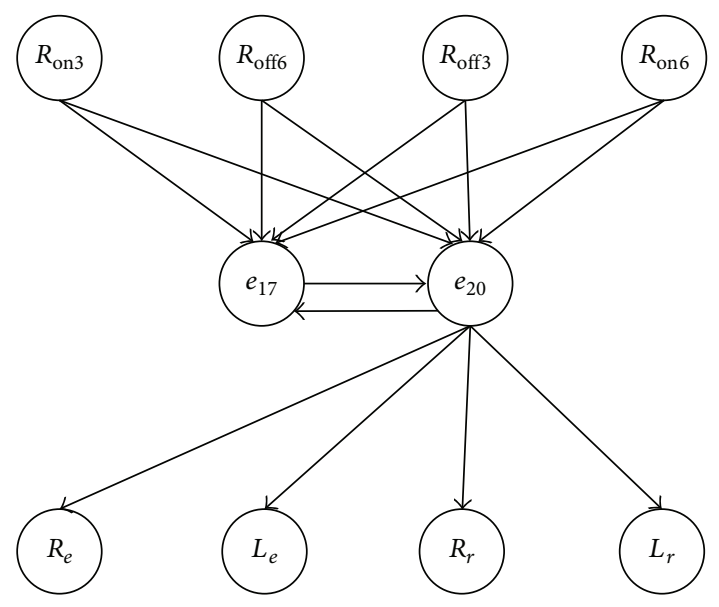

FIGURE 9: Directed graph of B-phase circuit.

$$
\begin{aligned}
& e_{46}=R_{r} f_{46}, \\
& f_{19}=f_{16}+f_{18}-f_{20}-f_{21}, \\
& f_{47}=\frac{1}{L_{r}} \int e_{47} d t,
\end{aligned}
$$

where $e_{i j}$ represents the effort variable of bond number $i j$ and $f_{i j}$ represents the flow variable of bond number $i j$. According to (4), the sum of $e_{17}$ and $e_{20}$ equals a constant value $e_{1}$, the DC input, such that the effort variables $e_{17}$ and $e_{20}$ are the interrelationship of cause and effect. The directed graph (DG) of B-phase circuit in number 1 inverter is shown as in Figure 9.

3.2.3. Building Bayesian Network. The first step is to establish the directed links between variables for a causal network by using the causality derived from the bond graph model of $\mathrm{CRH}_{5}$ traction system. Secondly, use intermediate variables 


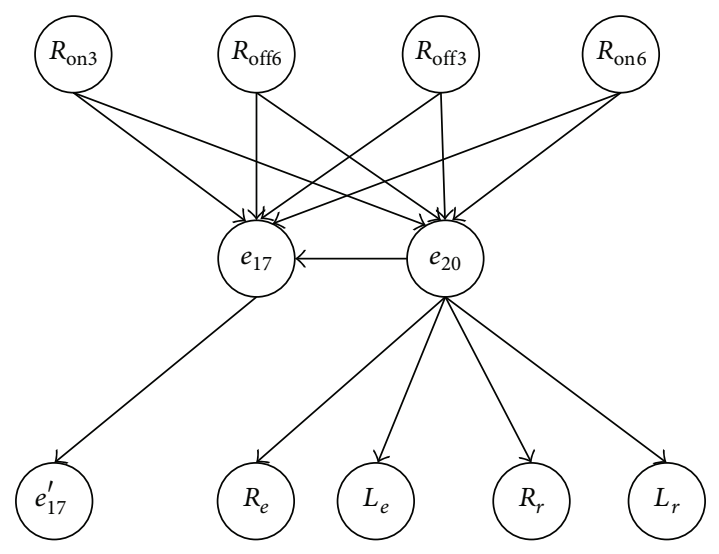

FIGURE 10: Bayesian network of B-phase circuit.

to obtain the conditional probability distributions (CPDs). Thirdly, specify the CPDs for each variable.

The directed graph model of B-phase circuit transforming from bond graph model is shown in Figure 9. Due to the reason that Bayesian network is a directed acyclic graph, some necessary simplifications are proposed for directed graph model having loops in [2]. The Bayesian network of B-phase circuit is shown in Figure 10, $R_{\text {on3 } 3}, R_{\text {off6 }}, R_{\text {off3 }}$, and $R_{\text {on6 } 6}$ are the root nodes corresponding to the components of B-phase circuit in equivalent diagram. $R_{e}, L_{e}, R_{r}$, and $L_{r}$ can indicate the behavior of the stator and rotor circuits which are the leaf nodes in the Bayesian network of the B-phase circuit. $e_{17}^{\prime}$ is the copied node of node $e_{17}$ according to the procedure in [2].

3.3. The Parameters of Bayesian Network. Different parameter learning algorithms are used to obtain the conditional probability table of the Bayesian network for complete and incomplete data sets. For complete data sets, Bayesian estimation algorithm is used for parameter estimation. It searches the parameter value with maximum posterior probability according to the a priori knowledge when the topological structure $S$ and training data set $D$ are known:

$$
P(\theta \mid D, S)=\frac{P(D \mid \theta, S) P(\theta \mid S)}{P(D \mid S)},
$$

where $\theta$ is a fixed unknown parameter and $P(\theta \mid S)$ is the prior probability of $\theta$ under the topological structure $S$. Let polynomial parameters $\theta_{1}, \theta_{2}, \ldots, \theta_{k}$ satisfy $\sum_{i=1}^{k} \theta_{i}=1$; then the posterior probability of the parameter can be represented by (6) when $P(\theta \mid S)$ is subject to Dirichlet distribution:

$$
\begin{aligned}
P(\theta \mid D, S) & =\operatorname{Dir}\left(\theta \mid \alpha_{1}, \alpha_{2}, \ldots, \alpha_{k}\right) \\
& =\frac{T(\alpha)}{\prod_{i=1}^{k} T\left(\alpha_{i}\right)} \prod_{i=1}^{k} \theta^{\alpha_{i}-1} .
\end{aligned}
$$

The formula of parameter estimation is as follow:

$$
\widehat{\theta}_{v \mid u}=\operatorname{Dir}\left(\alpha_{l}+N\left(x_{1}, u\right), \ldots, \alpha_{v k}+N\left(v_{k}, u\right)\right) .
$$

For incomplete data sets, the expectation maximization (EM) based iterative algorithm is used to compute the maximum likelihood probability of the network. Let initial value of the parameter be equal $\theta^{(0)}$. Modify the parameter constantly to achieve its maximum value of the maximum likelihood probability $E\left[\ln p\left(\begin{array}{l|l}Y \mid \theta\end{array}\right)\right.$, where $Y$ is the whole of the training sample.

The probability distribution expectation of data set $Y$ is represented as follows when the observable training sample $D$ and current $\theta$ are given:

$$
\begin{aligned}
Q\left(\theta^{(t)} \mid \theta\right) & =E\left[\ln p\left(Y \mid \theta^{(t)}\right) \mid \theta, D\right] \\
& =\sum_{l} \sum_{Z_{l}} \ln p\left(D_{l}, Z_{l} \mid \theta\right) p\left(Z_{l} \mid D_{l}, \theta^{(t)}\right) .
\end{aligned}
$$

Maximize the function $Q\left(\theta^{(t)} \mid \theta\right)$ by means of maximum likelihood estimation algorithm:

$$
\theta^{(t)}=\arg \max _{\theta^{(t)}} Q\left(\theta^{(t)} \mid \theta\right),
$$

where $D$ represents observable data set; $Z$ represents the data set which has not been observed; the whole training data $Y=$ $D \cup Z$.

3.4. Fault Prognosis Scheme. In this paper, fault prognosis scheme based on Bayesian network of B-phase circuit is designed to predict fault probability of stator or rotor circuit that may cause abnormal behaviors of overall system. The Bayesian network based fault prognosis is a kind of prediction mechanism using joint probability distribution to obtain the fault probability of child nodes, when network structure, fault probabilities of the root nodes, and conditional probability table of the other nodes are given. The causal variable analysis is main application of Bayesian network when the observed statues on any of the random variables are given. Conditional probability of unobserved modes is updated through belief propagation and inference can be made about the most probable status [32]. In the example of Figure 10, if variable $R_{e}$ is observed as true, the statues of $R_{\text {off6 }}$ can be inferred from this evidence such that the probability $P_{R_{\text {offi }} R_{e}}(a$, ture) is needed for this inference, and it can be quantified by marginalizing the joint distribution under the condition that the status of $R_{e}$ is known:

$$
\begin{aligned}
& P_{R_{\mathrm{off} 6} \mid R_{e}}(a, \text { true }) \\
& =\sum_{b} \sum_{c} \sum_{e} \sum_{f} \sum_{g} P\left(R_{\mathrm{off} 6}, R_{\mathrm{on} 3}, R_{\mathrm{off} 3}, R_{e}, R_{\mathrm{on} 6}, e_{20}, e_{17}\right) \\
& =\sum_{b} \sum_{c} \sum_{e} \sum_{f} \sum_{g} P_{R_{\mathrm{offi}}}(a) P_{R_{\mathrm{on} 3}}(b) P_{R_{\mathrm{off} 3} \mid R_{\mathrm{off} 6} R_{\mathrm{on} 3}}(a, b, c) \ldots,
\end{aligned}
$$

where $a, b, c, d, e, f$, and $g$ are the values of the nodes $R_{\text {off }}$, $R_{\mathrm{on} 3}, R_{\mathrm{off} 3}, R_{e}, R_{\mathrm{on} 6}, e_{20}$, and $e_{17}$. The difficulty of this approach is that, as the number of nodes grows big, precise calculation of new condition probability becomes an NP-hard problem. Due to the reason above, the polytree propagation algorithm, 
a Monte Carlo based approximate reasoning algorithm, is adopted in this paper. According to the Bayesian network in Figure 10, the fault probabilities of each node are as follows:

$$
\begin{gathered}
P\left(e_{20}=f\right) \\
=\sum_{R_{\mathrm{on} 3,6}, R_{\mathrm{off} 3,6}} P\left(e_{20}=f \mid R_{\mathrm{on} 3}, R_{\mathrm{off} 6}, R_{\mathrm{off} 3}, R_{\mathrm{on} 6}\right) \\
\cdot P\left(R_{\mathrm{on} 3}, R_{\mathrm{off} 6}, R_{\mathrm{off} 3}, R_{\mathrm{on} 6}\right), \\
P\left(e_{17}=f\right) \quad P\left(e_{17}=f \mid R_{\mathrm{on} 3}, R_{\mathrm{off} 6}, R_{\mathrm{off} 3}, R_{\mathrm{on} 6}, e_{20}\right) \\
=\sum_{R_{\mathrm{on} 3,6}, R_{\mathrm{off} 3,6}, e_{20}} \quad P\left(R_{\mathrm{on} 3}, R_{\mathrm{off} 6}, R_{\mathrm{off3}}, R_{\mathrm{on} 6}, e_{20}\right), \\
P\left(R_{e}=f\right) \sum_{R_{\mathrm{on} 3,6}, R_{\mathrm{off} 3,6}, e_{20}} P\left(R_{e}=f \mid R_{\mathrm{on} 3}, R_{\mathrm{off} 6}, R_{\mathrm{off} 3}, R_{\mathrm{on} 6}, e_{20}\right) \\
\quad P\left(R_{\mathrm{on} 3}, R_{\mathrm{off} 6}, R_{\mathrm{off} 3}, R_{\mathrm{on} 6}, e_{20}\right) .
\end{gathered}
$$

\section{Results and Discussions}

Firstly, a bond graph model of $\mathrm{CRH}_{5}$ traction system is formed in the environment of 20-SIM. When open-circuit fault happens on IGBT $v_{3}$, load current $i_{b}$ loses its positive half wave, which may cause the motor stalling. When shortcircuit fault happens on IGBT $v_{3}, i_{b}$ increases rapidly, such that short circuit on IGBT which may cause catastrophic failure on stator or rotor circuit is the fault we studied in simulation section.

In this section, 10000 sets of data produced by experiments are divided into 10 groups, where 600 sets of data are for training and 400 sets of data are for test in each group. According to [33], the fault distribution in electric drive system is as follows: power devices (37\%), capacitors $(20 \%)$, inductors $(5 \%)$, resistors $(2 \%)$, connectors $(15 \%)$, gate drives $(16 \%)$, and others (5\%). What is more, in [33], the fault probabilities of IBGT open-circuit fault (37\%) and shortcircuit fault (43\%) are also given. Regarding the complete data from experiments as the training sample of parameter learning, compute the conditional probability of intermediate nodes and leaf nodes by maximum likelihood estimation. The conditional probability table is shown in Table 2 , where $n, f$ represent normal nodes and faulty nodes, respectively.

Predict the fault probability of stator or rotor circuit when short-circuit fault of IGBT components in B-phase inverter circuit happens. According to Pearl's polytree propagation algorithm used for joint probability reasoning, the prediction results on fault probability of stator and rotor circuit through 10000 sets of complete data are shown in Figures 11 and 12.

In modern engineering systems, some values in the measurement data set are missing such that EM (expectation maximization) method, a well-known parameter estimation algorithm, is used for probabilistic reasoning, combining with
TABLE 2: Conditional probability table of parameter learning.

\begin{tabular}{lc}
\hline Conditional probability & Value \\
\hline$P\left(R_{\text {on } 3}=f\right)$ & 0.137 \\
$P\left(R_{\text {off6 }}=f\right)$ & 0.159 \\
$P\left(R_{\text {off3 }}=f\right)$ & 0.159 \\
$P\left(R_{\text {on6 }}=f\right)$ & 0.137 \\
$P\left(e_{20}=f \mid R_{\text {on3 }}=n, R_{\text {off6 }}=n, R_{\text {off3 }}=n, R_{\text {on6 }}=n\right)$ & 0 \\
$P\left(e_{20}=f \mid R_{\text {on3 }}=f, R_{\text {off6 }}=n, R_{\text {off3 }}=n, R_{\text {on6 }}=n\right)$ & 0.95 \\
$P\left(e_{20}=f \mid R_{\text {on3 }}=n, R_{\text {off6 }}=f, R_{\text {off3 }}=n, R_{\text {on6 }}=n\right)$ & 0.95 \\
$P\left(e_{20}=f \mid R_{\text {on3 }}=n, R_{\text {off6 }}=n, R_{\text {off3 }}=f, R_{\text {on6 }}=n\right)$ & 0.95 \\
$P\left(e_{20}=f \mid R_{\text {on3 }}=n, R_{\text {off6 }}=n, R_{\text {off3 }}=n, R_{\text {on6 }}=f\right)$ & 0.95 \\
$P\left(e_{20}=f \mid R_{\text {on3 }}=f, R_{\text {off6 }}=f, R_{\text {off3 }}=n, R_{\text {on6 }}=n\right)$ & 0.98 \\
$P\left(e_{20}=f \mid R_{\text {on3 }}=f, R_{\text {off6 }}=n, R_{\text {off3 }}=n, R_{\text {on6 }}=f\right)$ & 0.92 \\
$P\left(e_{20}=f \mid R_{\text {on3 }}=n, R_{\text {off6 }}=f, R_{\text {off3 }}=f, R_{\text {on6 }}=n\right)$ & 0.92 \\
$P\left(e_{20}=f \mid R_{\text {on3 }}=n, R_{\text {off6 }}=n, R_{\text {off3 }}=f, R_{\text {on6 }}=f\right)$ & 0.98 \\
$P\left(R_{e} \mid e_{20}\right)$ & 0.511 \\
$P\left(L_{e} \mid e_{20}\right)$ & 0.511 \\
$P\left(R_{r} \mid e_{20}\right)$ & 0.370 \\
$P\left(L_{r} \mid e_{20}\right)$ & 0.370 \\
\hline
\end{tabular}

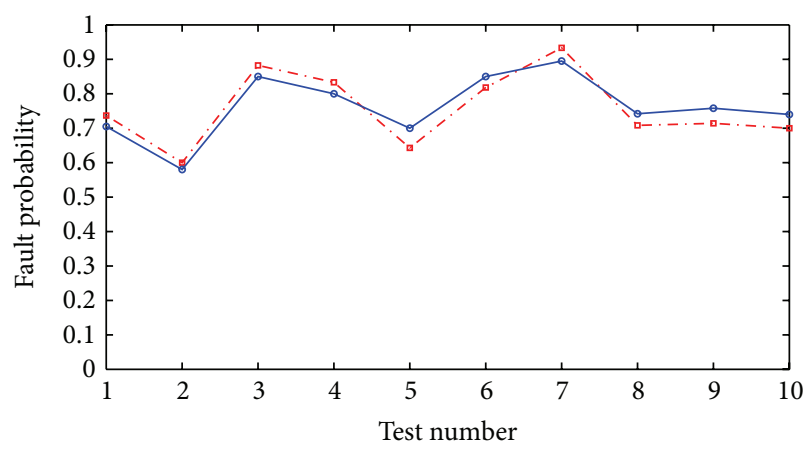

$\rightarrow-$ Predicted probability

$\because$ Actual probability

FIGURE 11: Fault prognosis of stator circuit.

Pearl's polytree propagation algorithm. Each iteration step of EM algorithm contains two steps: the E-step (expectation) and the M-step (maximization). The procedure alternates between the two steps until convergence is achieved. It should be pointed out that the search speed will slow down when EM algorithm comes close to its convergence point.

In this simulation, 10000 sets of data are hidden randomly by $20 \%$ to imitate the data missing. The remaining sets are divided into 10 groups, where 600 sets of data are for training and 200 sets of data are for test in each group. The conditional probability table of each node can be obtained by EM algorithm. Predict the fault probability of leaf nodes by Pearl's polytree propagation algorithm when prior probabilities of the root nodes are given. In Figures 13 and 14, the prediction results of stator or rotor circuit under incomplete data can also verify effectiveness of the proposed approach. 


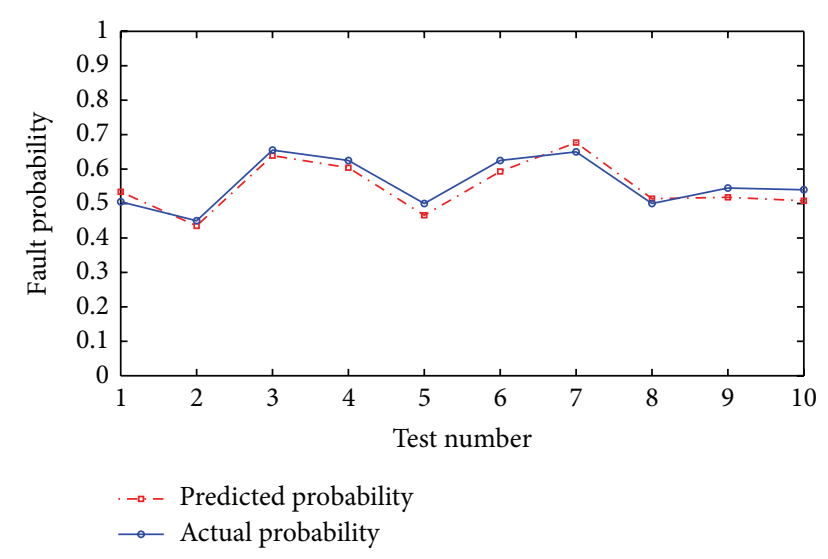

FIGURE 12: Fault prognosis of rotor circuit.

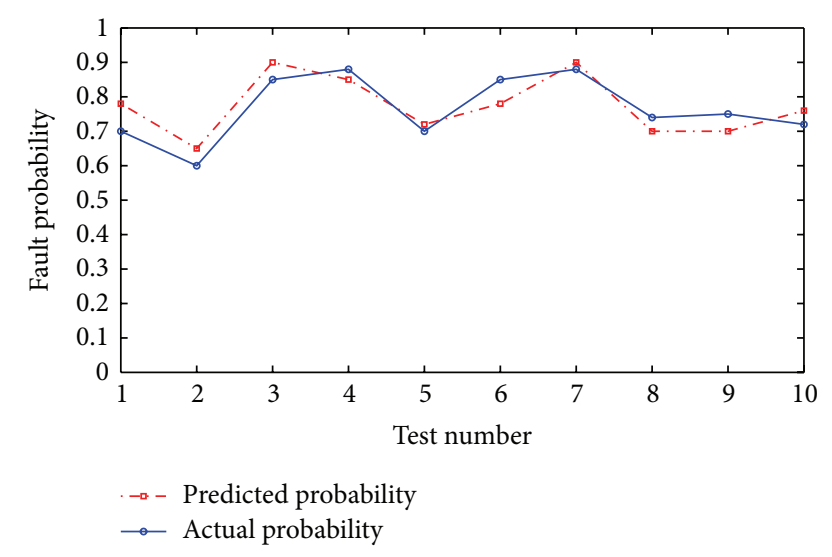

FIGURE 13: Fault prognosis of stator circuit.

\section{Conclusion}

According to the schematic diagram of $\mathrm{CRH}_{5}$ traction system, a bond graph based model of inverter circuit and threephase AC motor is built. Then, a bond graph and Bayesian network based fault prognosis approach is proposed to predict the fault probability of stator and rotor circuit, when the prior fault probabilities of IGBT components are given. The bond graph based model of $\mathrm{CRH}_{5}$ traction system is used for building the Bayesian network, which can solve the problem of constructing an accurate Bayesian network structure in practice. Different parameter learning algorithms, such as Bayesian estimation and EM algorithm, are adopted to determine the conditional probability table of the Bayesian network for complete and incomplete data sets. The fault probabilities of leaf nodes (stator and rotor circuit) can be predicted by joint probability reasoning through Pearl's polytree propagation algorithm. The simulation results can verify its effectiveness in fault prognosis for both complete and incomplete data sets. Our future works lie in the following: (1) the bond graph modeling of junction parts should be considered; (2) some new bond graph modeling approaches of power switching device (IGBT) can be adopted in our future work to achieve a more accurate modeling.

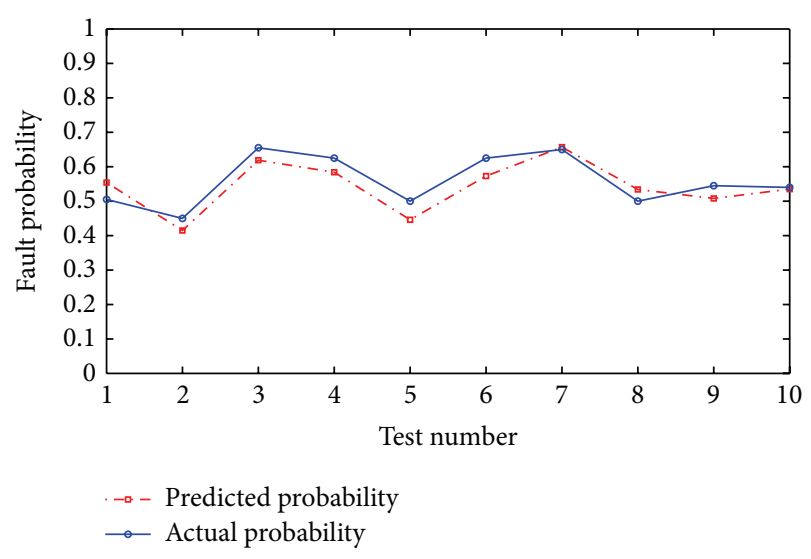

FIGURE 14: Fault prognosis of rotor circuit.

\section{Conflict of Interests}

The authors declare that there is no conflict of interests regarding the publication of this paper.

\section{Acknowledgments}

This work was supported by National Natural Science Foundation of China (61490703, 61374141 and 61304112), Funding of Jiangsu Innovation Program for Graduate Education KYLX_0280, and the Fundamental Research Funds for the Central Universities (NE2014202).

\section{References}

[1] G. Vachtsevanos, F. L. Lewis, M. Roemer, A. Hess, and B. Wu, Intelligent Fault Diagnosis and Prognosis for Engineering Systems, John Wiley \& Sons, New York, NY, USA, 2007.

[2] N. Lu, K. He, and B. Jiang, "A fault prognosis method using Bayesian network," Journal of Southeast University, vol. 42, no. A01, pp. 87-91, 2012.

[3] L.-J. Xu, H.-J. Wang, and B. Long, "Fault prediction of complex systems based on Bayesian network," Systems Engineering and Electronics, vol. 30, no. 4, pp. 780-784, 2008.

[4] F. V. Jensen, Bayesian Networks and Decision Graphs, Springer, New York, NY, USA, 2001.

[5] M. Singh and M. Valtorta, "Construction of Bayesian network structures from data: a brief survey and an efficient algorithm," International Journal of Approximate Reasoning, vol. 12, no. 2, pp. 111-131, 1995.

[6] A. Okutan and O. T. Yıldı, "Software defect prediction using Bayesian networks," Empirical Software Engineering, vol. 19, no. 1, pp. 154-181, 2014.

[7] J. Lin, M. Asplund, and A. Parida, "Reliability analysis for degradation of locomotive wheels using parametric Bayesian approach," Quality and Reliability Engineering International, vol. 1, no. 1, pp. 1-11, 2013.

[8] H. Paynter, Analysis and Design of Engineering Systems, MIT Press, Cambridge, Mass, USA, 1961.

[9] G. Dauphin-Tanguy, Les Bond Graphs, HERMES Science, Paris, France, 2007. 
[10] J. U. Thoma, Introduction to Bond Graphs and Their Applications, Pergamon Press, New York, NY, USA, 1975.

[11] N. Banerjee and R. Karmakar, "Bond graph modeling of rail wheelset on curved track," Simulation, vol. 83, no. 10, pp. 695706, 2007.

[12] G. Gandanegara, X. Roboam, B. Sareni, and G. Dauphin-Tanguy, "Bond-graph-based model simplification for system analysis: application to a railway traction device," Proceedings of the Institution of Mechanical Engineers, Part I: Journal of Systems and Control Engineering, vol. 220, no. 7, pp. 553-571, 2006.

[13] J. Lozano, J. Felez, and J. D. Sanz, "Comparative study of railway drive systems using bond-graph," in Proceedings of the Spring Simulation Multiconference, April 2010.

[14] J. Kim and M. D. Bryant, "Bond graph model of a squirrel cage induction motor with direct physical correspondence," Journal of Dynamic Systems, Measurement, and Control, vol. 122, no. 3, pp. 461-469, 2000.

[15] D. Du, B. Jiang, P. Shi, and H. Karimi, "Fault detection for continuous-time switched systems under asynchronous switching," International Journal of Robust and Nonlinear Control, vol. 24, no. 11, pp. 1694-1706, 2014.

[16] D. Du, B. Jiang, and P. Shi, "Fault detection for discrete-time switched systems with intermittent measurements," International Journal of Control, vol. 85, no. 1, pp. 78-87, 2012.

[17] Z. Mao, B. Jiang, and P. Shi, "Fault detection for a class of nonlinear networked control systems," International Journal of Adaptive Control and Signal Processing, vol. 24, no. 7, pp. 610$622,2010$.

[18] X.-Z. Gao, H. Xu, X. Wang, and K. Zenger, "A study of negative selection algorithm-based motor fault detection and diagnosis," International Journal of Innovative Computing, Information and Control, vol. 9, no. 2, pp. 875-901, 2013.

[19] Y. Kourd, D. Lefebvre, and N. Guersi, "Fault diagnosis based on neural networks and decision trees: application to DAMADICS," International Journal of Innovative Computing, Information and Control, vol. 9, no. 8, pp. 3185-3196, 2013.

[20] P. J. Mosterman and G. Biswas, "Diagnosis of continuous valued systems in transient operating regions," IEEE Transactions on Systems, Man, and Cybernetics Part A: Systems and Humans., vol. 29, no. 6, pp. 554-565, 1999.

[21] K. Medjaher, A. K. Samantaray, B. O. Bouamama, and M. Staroswiecki, "Supervision of an industrial steam generator. Part II: online implementation," Control Engineering Practice, vol. 14, no. 1, pp. 85-96, 2006.

[22] A. K. Samantaray, K. Medjaher, B. O. Bouamama, M. Staroswiecki, and G. Dauphin-Tanguy, "Diagnostic bond graphs for online fault detection and isolation," Simulation Modelling Practice and Theory, vol. 14, no. 3, pp. 237-262, 2006.

[23] A. K. Samantaray and S. K. Ghoshal, "Bicausal bond graphs for supervision: from fault detection and isolation to fault accommodation," Journal of the Franklin Institute, vol. 345, no. 1, pp. 1-28, 2008.

[24] X. Zhang and K. A. Hoo, "Effective fault detection and isolation using bond graph-based domain decomposition," Computers and Chemical Engineering, vol. 35, no. 1, pp. 132-148, 2011.

[25] M. Yu and D. Wang, "Model-based health monitoring for a vehicle steering system with multiple faults of unknown types," IEEE Transactions on Industrial Electronics, vol. 61, no. 7, pp. 3574-3586, 2014.

[26] S. Zhang, CRH5 Series High-Speed Railway Vehicle, China Railway Publishing, Beijing, China, 2008.
[27] M. A. Djeziri, R. Merzouki, B. Ould Bouamama, and G. Dauphin-Tanguy, "Bond graph model based for robust fault diagnosis," in Proceedings of the American Control Conference (ACC '07), pp. 3017-3022, July 2007.

[28] A. K. Samantaray and B. O. Bouamama, Model-Based Process Supervision: A Bond Graph Approach, Springer, Berlin, Germany, 2008.

[29] C. B. Low, D. Wang, S. Arogeti, and J. B. Zhang, "Causality assignment and model approximation for hybrid bond graph: fault diagnosis perspectives," IEEE Transactions on Automation Science and Engineering, vol. 7, no. 3, pp. 570-580, 2010.

[30] C. H. Lo, Y. K. Wong, and A. B. Rad, "Bond graph based Bayesian network for fault diagnosis," Applied Soft Computing Journal, vol. 11, no. 1, pp. 1208-1212, 2011.

[31] K. Dejaeger, T. Verbraken, and B. Baesens, "Toward comprehensible software fault prediction models using bayesian network classifiers," IEEE Transactions on Software Engineering, vol. 39, no. 2, pp. 237-257, 2013.

[32] L. Yang and J. Lee, "Bayesian Belief Network-based approach for diagnostics and prognostics of semiconductor manufacturing systems," Robotics and Computer-Integrated Manufacturing, vol. 28, no. 1, pp. 66-74, 2012.

[33] S. Yang, A. Bryant, P. Mawby, D. Xiang, L. Ran, and P. Tavner, "An industry-based survey of reliability in power electronic converters," IEEE Transactions on Industry Applications, vol. 47, no. 3, pp. 1441-1451, 2011. 


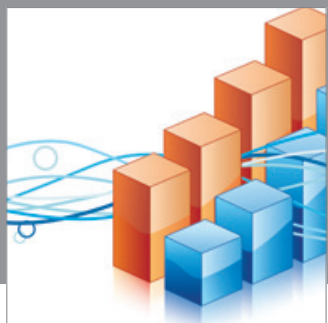

Advances in

Operations Research

mansans

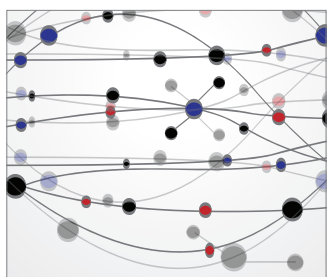

The Scientific World Journal
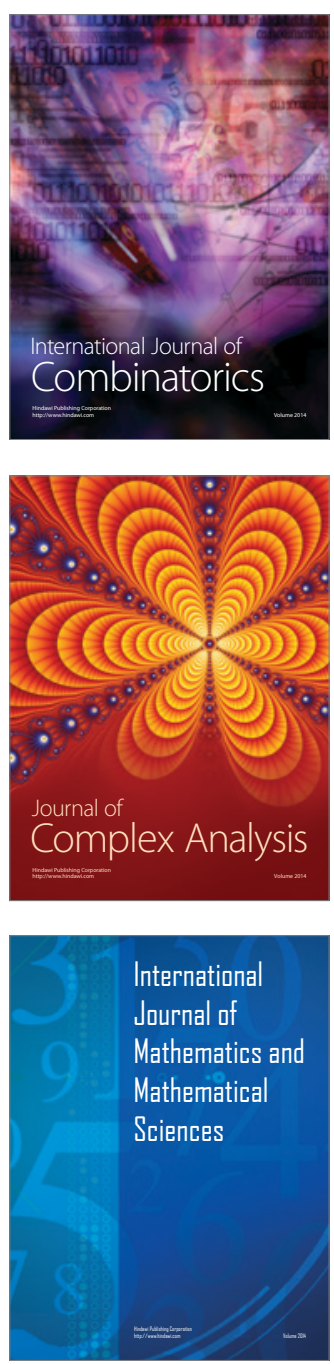
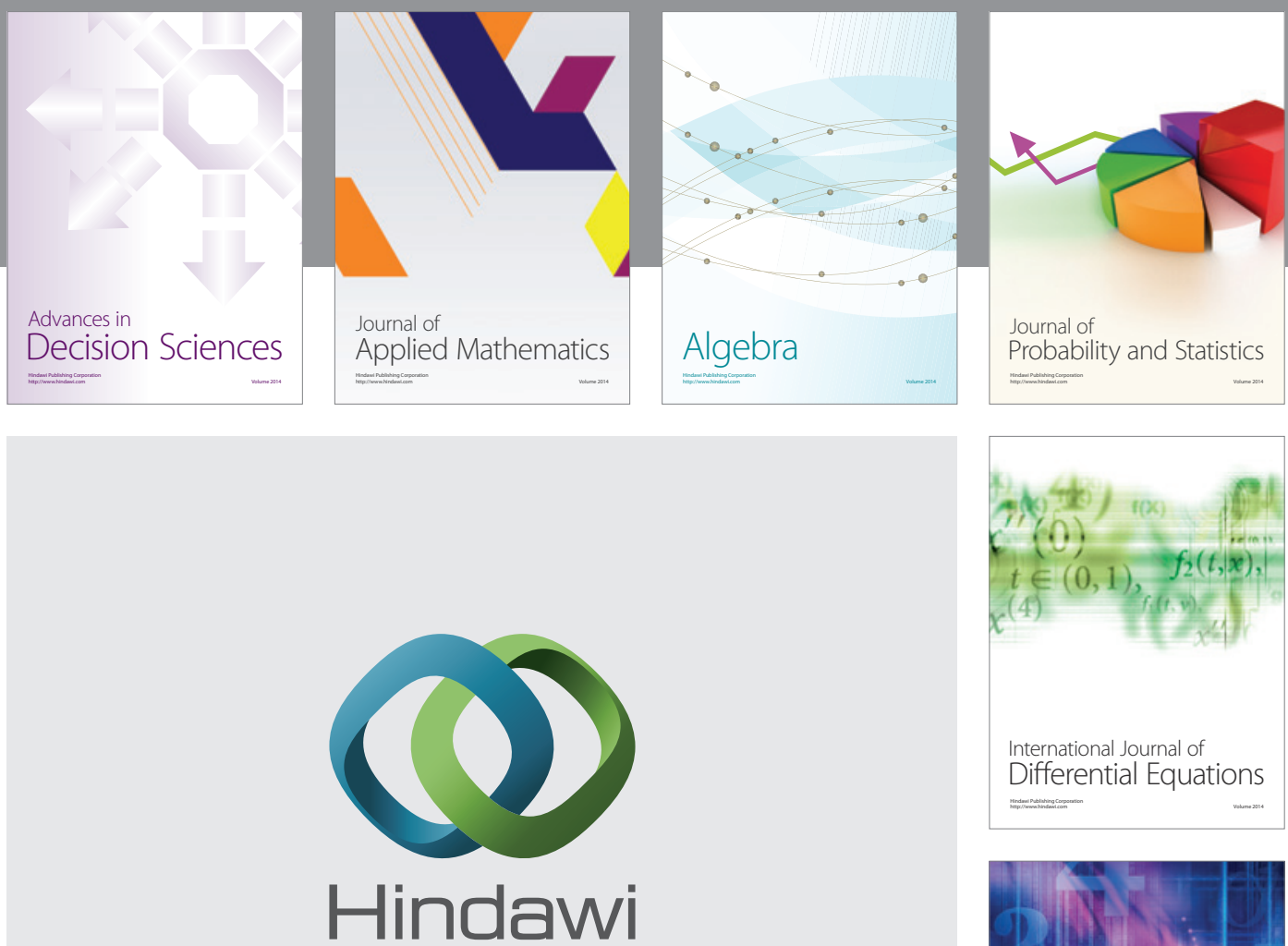

Submit your manuscripts at http://www.hindawi.com
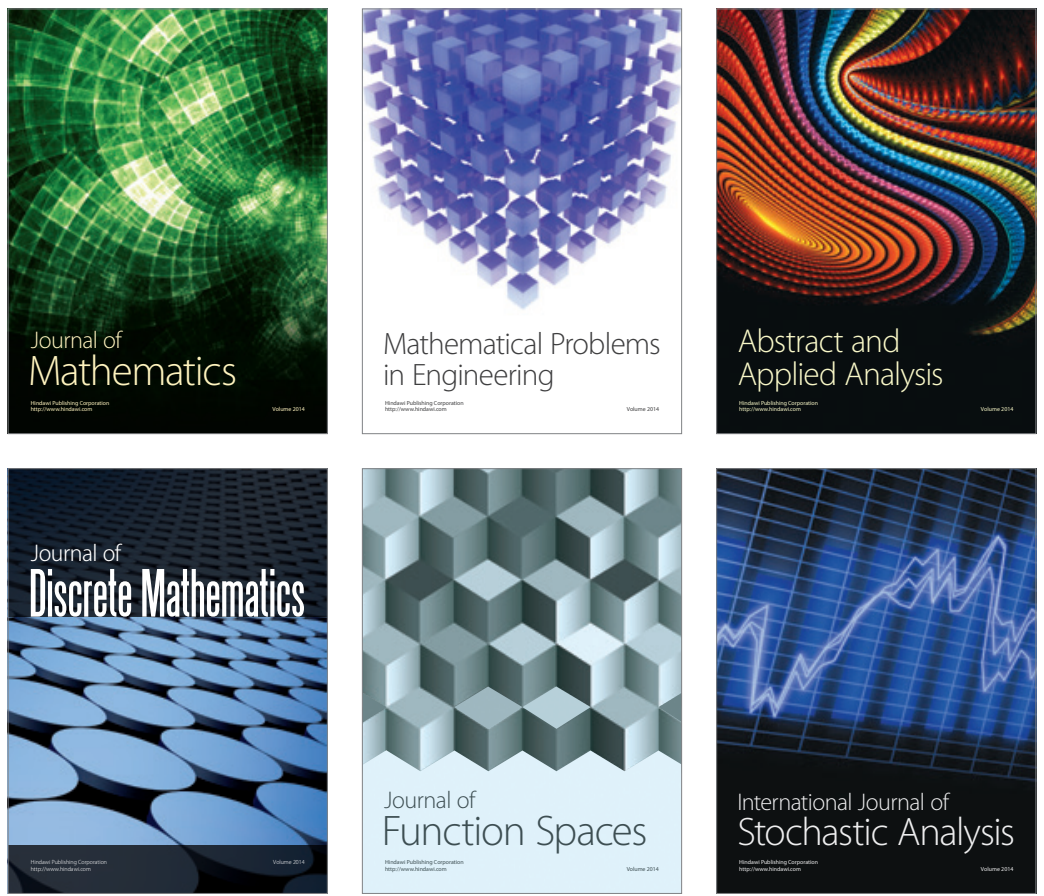

Journal of

Function Spaces

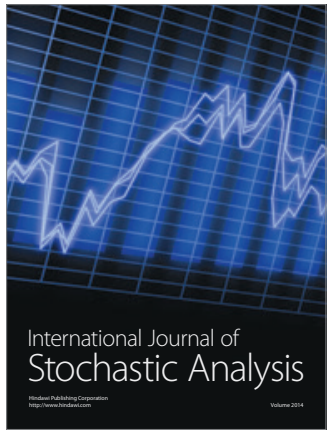

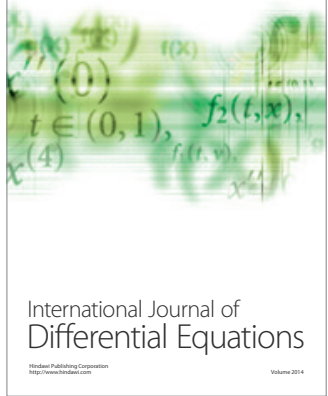
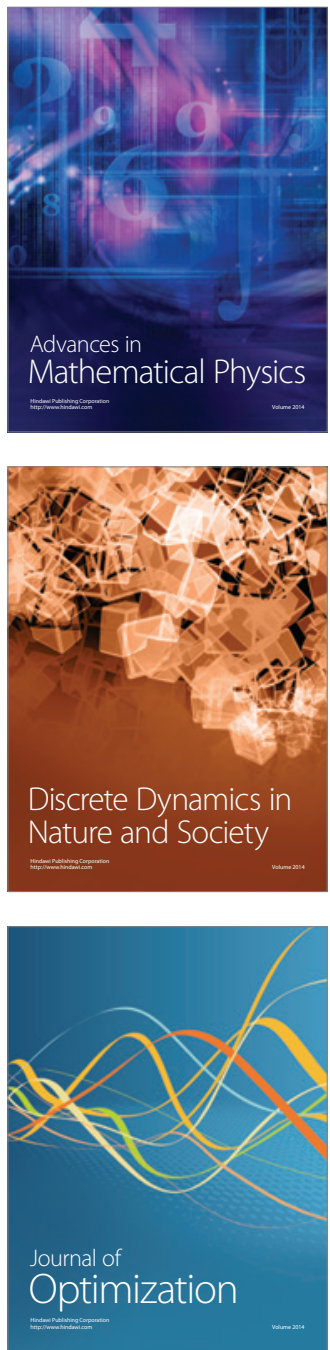